\title{
Design of Electric Emergency repair system and Its Security Based on Mobile Terminal
}

\author{
Yuan Xie ${ }^{1,2, a}$, Shaomin Zhang ${ }^{1, b}$, Baoyi Wang ${ }^{1, c}$ \\ ${ }^{1}$ School of Control and Computer Engineering, North China Electric Power University, Baoding, \\ 071003, China, \\ ${ }^{2}$ State Grid Panji Power Supply Company, Huainan, 232000

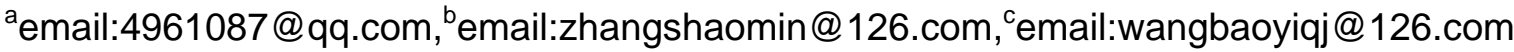

\section{Keywords: Mobile Internet; Electrical Repair; Mobile Terminal; Security}

\begin{abstract}
With the rapid development of mobile Internet technology, a electrical emergency repair system is designed based on mobile terminal. The security mechanism of the system is also designed following the principle of appropriate protection, and aim at security defects of mobile internet technological and the high security requirement of power system. The system architecture includes three layers, Intranet application layer, Mobile security access layer and terminal layer. By improving the SSL protocol, the mobile security access channel based on improved SSL protocol is constructed to realize the application system security.
\end{abstract}

\section{Introduction}

With the continuous development of power system, the scale of the power grid is expanding, the power users increase year by year, protecting the user's normal power supply is the basics of providing quality service. But a variety of electrical equipment that are distributed in the complex external environment may be malfunction at any time, causing a large area of power outages, not only to the local people's lives a great deal of inconvenience, but also cause huge economic losses. Therefore, electrical emergency repair has become a key factor in whether to provide high-quality power supply services. Therefore, many electrical companies are developing the electrical emergency repair system actively.

In 2011, a company in Shenyang China realized the vehicle path analysis function, positioning function and navigation function of electrical repair mobile terminal, and communication function and data transmission function of terminal etc. . Using the embedded GIS component to carry out secondary development, which makes the map operation on the PDA is very simple, reduces the system's memory consumption and improves the system performance[1]. And a real-time electrical repair service system based on PDA was developed in Zhejiang, the system realized the informational workflow of electrical system fault processing feedback. The system collects the information of electrical system failure in real time, and promptly notifies the front line repair service personnel through PDA and wireless data services (CDMA). Repair personnel feedback the handling information by PDA after they dealt with the malfunction, and uploaded the results to the 95598 system through the PDA[2].In 2013,a city in Shanxi designed a electrical dispatch scheduling system by fully using global positioning technology, GPRS technology, geographic information technology; The system receives the positioning information through GPS, and the final data's by short message were sent to the monitoring center through GPRS network, the vehicle terminal can receive the control commands sent by the monitoring center too; At present, the system has been successfully used in electrical repair scheduling command center [3]. In 2014, a company in Jiangsu designed an 
electrical emergency repair system by GIS, that the system can reasonable schedule electrical emergency repair, repair vehicles and repair resources, and designed the location of the fault and repair vehicle and the optimal path algorithm, the system uses WebGIS architecture of B/S model [4].

With the rapid development of Internet applications, open and shared development model makes the Internet a distribution center of new business, new ideas and a mass of information. For Internet applications, the ability of telecommunications to secure communications is the greatest attraction to the Internet. The telecommunication network needs the Internet service mode. Represented by smart phones, tablets, mobile Internet devices give the user subversive change of experience and the use of the habits. If taking various mobile intelligent terminal with dexterity, convenience, efficiency, sensitivity and other characteristics as a carrier, a large number of power on-line monitoring, mobile operation and maintenance management equipment will greatly enhance the power production and operation and maintenance information and modernization. Therefore, mobile communication technology has become an indispensable technology to build power application system. To this end, China's electric power industry carry out a series of mobile information applications attempt within the system since 2010,and the grid enterprise relied on mobile Internet has made a deep exploration especially in the field operations. But it is worth noting that the mobile Internet provides sophisticated management and quality services for the company's business processing but also faces a number of security challenges. On the one hand, these challenges come from the traditional Internet security threats. On the other hand, they are from the new security issues that mobile Internet brings due to its own characteristics.

There are several important roots of Internet security issues. First, the network transparent to the user. Users can obtain the IP address of any important network node and initiate vulnerability scanning and attack. The network topology can be easily obtained by the attacker. The attacker can intercept and modify the data transmitted in the network, so the user data security is not guaranteed. Second, the user opaque to the network. Authentication is not strict, and therefore a large number of users without a strict authentication mechanism can access the network. The network can not control and knows nothing about the terminal security capabilities and security status. User address can be forged, and can not be reliable traceability. In short, the integration of mobile and the Internet results in the loss of the traditional mobile network security advantage, and the rest is only a few features such as rigorous authentication, behavior traceability.

As the grid companies gradually build and improve the mobile application platform, many core business has gradually expanded to the mobile platform, and remote data interaction becomes more and more frequent. When the mobile terminal interacts with the back-end service, the root of threatening the network operation and data security is the "single-machine double-network" status of accessing the mobile Internet and the enterprise network at the same time. These problems are embodied in the following aspects.

The mobile terminals may have their own security vulnerabilities, which will make the terminal more vulnerable where the corporation data files have already been in lack of protection before using of mobile terminals. During the transmission, data are in risk of interference, intercept and tampering, the intranet services are directly orient to the incoming outside terminal connections. Once the mobile terminals successfully connect to electrical information intranet, they are assumed as a trusted user inside this electrical information intranet and can get the access to the intranet resources. The security of the intranet cannot be assured once the terminals in the connection and data transmission process with intranet are under attack.

The electrical systems should be highly reliable, which is showed as the assurance of protecting classified data from leakage while the system allows all kinds of discrete access subjects can connect 
to the electrical information network securely and credibly. However, since the security problems have not been solved yet, the mobile terminal based electrical information system cannot be used immediately even though there are huge application demands on this kind of systems. So, researches on the security plan for this systems have been brought into the spotlight.

To solving some of the shortcomings of current technologies, in this paper, a design plan for the mobile terminal based electrical grid repair assistance system and its security mechanism is presented, in order to increase the degree of imformatization and modernization in produce and operation process, while assuring the security of electrical corporation intranet.

\section{Design Of Electric Emergency Repair System Mobile Terminal Based}

According to the on-site requirement of power system, the terminal based electric emergency repair system have these functions as below.

"Weave The Net" Function. Considering every APP users of this system as a 'spider point', during the daily work of these users the APP can automatically(manually) recording the track of work activities (including texts and images) according configuration settings. In this way, accumulatively, the social electrical information network can be created, which is available 24/7 and can be looked up by the user in the future.

Navigating Function. This APP can perform navigation in the electrical information network created by multiple spider points.

Suggesting Function. This APP can automatically provide some travel suggestions by analyzing the information from frontline users and various social announcements. In a travel, this APP can provide information about the pre-set road plan such as the possible closed road because of maintenance, possible congestion on narrow streets, possible crowded area where a market exists and also the number of traffic lights on the travel plan, speed limits and estimated arrival time, etc. This function will help the user to avoid the traffic congestion as much as possible, which in turn reduces the compliant rate.

Lookup Function. Users can lookup the information about power line, pole, electricity meter and house substation (including photos, type serial number, service time, spare parts). This function provides the basic information of working site, saving a lot of time and energy for service members and preparation of spare parts.

Position Indication Function. This APP can present and share the user's location. This function enables power supply services collaboration and provides the possibility of management informationization and refinement.

Sharing Function. This APP can share the site photos and some descriptions to the online album, then other users can synchronize these photos and have a review. This function constructs a technological bridge between the working site and distance office.

The basic configuration requirement to the users is, operating system Android 2.3 or above, smart phone that has Internet access. That is, the mobile phone terminals which can access the Internet via cell networks (like 2G, 3G, E3G).

\section{Security Design For The System}

The security of mobile internet can be a complex systematic problem, since the security of multiple components of this system should be taken into consideration, in which at least the security of terminal devices, mobile network, business application is included. As a consequence, any part that lack of protection will become into the weak point of the system that makes the whole system 
vulnerable. Considering this kind of technical complexity as security defect, this problem has forbidden the wider application of mobile internet. Many groups have commenced researches on this area and a number of papers on this topic have been published [5-8]. As for this paper, With respect to the current application and future development of mobile internet in electrical grid corporations, taking the moderate protection as principle and by refining the meticulous control methods of Mobile Informationization Business, the group of this paper has designed a security plan for the specific mobile internet system, in order to protect the system from application system sabotage, business data loss and corporation data leakage, then ensure the stability and security of this system.

In security designation for a particular system, instead of trying to fulfill all of the security demands, premised upon the assurance of system security, the limitations in real condition such as the operability and economic constraint should be considered, which is called practical and reliable moderate protect principle of design.

In the text below, four different protection plan has been suggested for different situation, every plan has its own advantages and can be applied to the specific system independently to act as system security protection mechanism.

The Figure1 shows the Electrical Repair Assistance System based on mobile terminal. The structure of this system consists of 3 layers which are intranet application layer, mobile terminal secure connection layer and terminal layer.
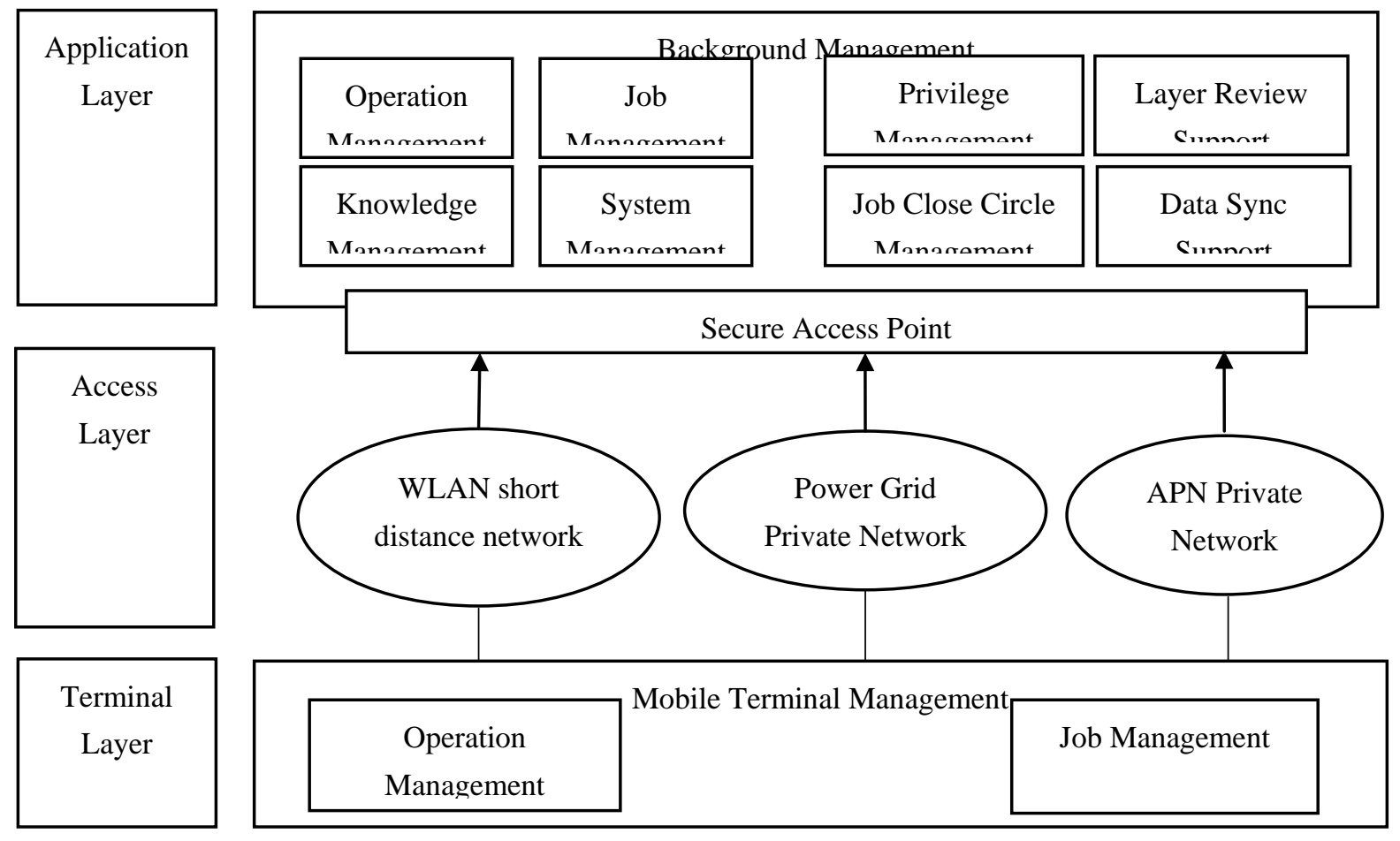

Fig1 The Structure of mobile terminal based Electrical Repair Assistance System

Intranet Application Layer. Resides in intranet and have direct access to intranet data. This layer usually supported by Windows operating system and the security reinforcements on operating system, database and application system have already been applied, which in turn has already met the requirements of intranet information system security.

Mobile Terminal Secure Connection Layer. This layer is responsible for two functions: network transmission and secure connection.

The network transmissions can be implemented by multiple technologies, which have their own advantages: 
WLAN Wireless Network. Which is implemented by wireless communication technology. This technology allows users to establish a long distance wireless connection with data network but with inadequate security.

APN Wireless Private Connection Technology. By using the APN/VPDN technology, this technology provides Mobile Informationization Business access to industry customers via GPRS/WCDMA network. By using this technology, the mobile terminal users can perform data exchanges with corporation's private network wirelessly, which shows the possibility to establish a secure corporation private network in national range that combined with fixed and mobile devices. This kind of technology provides a highly reliable, highly secure and highly administrable private network, enables the fast virtual network establishment for corporations which solved the security problems in public networks at a relatively high expense.

Private Network Of Electrical Grid Technology. It is not enough for the new smart electrical grid business to transfer data on a borrowed public network because the smart electrical grid requires a high-bandwidth, real-timing, secure and reliable transmission solution, especially when at the peak time of using the communication devices, there is a high risk to encounter a "traffic jam" at a public network. Meanwhile, in a public network, the power distribution or power consumption devices cannot be effectively monitored by the electrical grid schedule center. This kind of "blind" management of devices causes a low reliability power transportation and low efficiency electrical grid operation, which tend to become the bottleneck of electrical grid communication. The effective solution is to establish and use the wireless private network of electrical grid.

$230 \mathrm{MHz}$ band is the national radio management committee for the energy, military, meteorological, earthquake, water conservancy, mining, light industry and other industries as telemetry, remote control, data transmission and other services using the band, Which allocated to the Department of Energy for the national power load monitoring system 40 frequency points. The bandwidth $230 \mathrm{MHz}$ band is $12 \mathrm{MHz}$. The power special network technology based on discrete spectrum aggregation, spectrum sensing, all IP network architecture design and other advanced technologies is to play the power of a dedicated frequency band in the interference-free, adjacent frequency interference source less, the propagation loss is small, covering ability, the weather on the signal transmission performance impact of small features to build power wireless private network. At present, the technology is still developing, the pilot application, but the use of the network there must be a cost problem, which is the system design program must be considered factors

Figure 2 is the security design of emergency repair system based on the mobile terminal.

This figure describes the factors to consider when selecting and designing a security scenario. The biggest problem in security is how to determine the degree of security, at different levels, security has a different meaning. To fully consider the objective conditions of the scene, a clear system should focus on protection of the part, rather than blindly pursue the realization of all security needs. As far as possible both to ensure safety, but also take into account the operability and economy, in a practical, reliable and appropriate protection of the principles of design. 


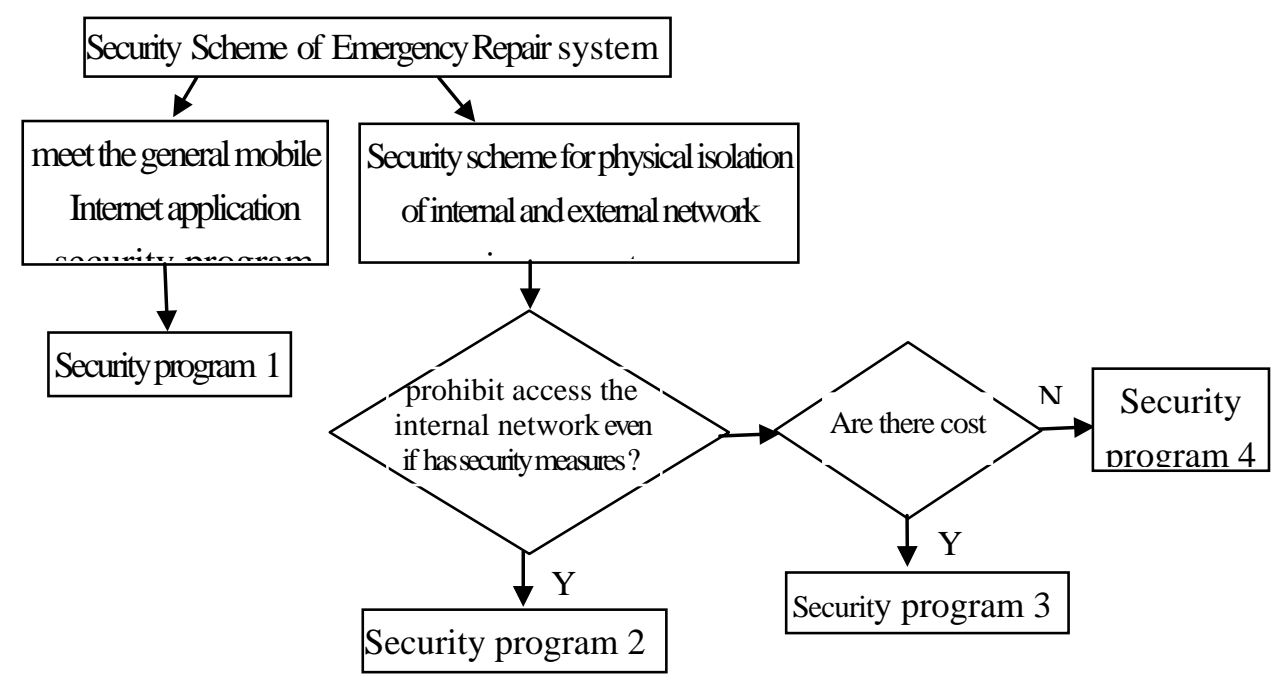

Figure2 Design of Safety Scheme for Emergency Repair System Based on Mobile Terminal The architecture is based on the introduction of SSL mobile security architecture. Extend the SSL VPN gateway to the mobile security access layer, and design a mobile security access point in this layer, to extend the traditional SSL handshake process based on the traditional SSL handshake method, to certify the client CA certificate, Legitimacy authentication and user authentication are placed in the final stage of the handshake to create dedicated authentication tunnels to support authentication of device authenticity, user identity, and security classification of different networks.

Terminal layer. The security access terminal layer comprises a mobile terminal and is mainly composed of security software and hardware modules.

Client user authentication method is to equipment, users and SIM card ternary binding to ensure that the special plane dedicated.

\section{Conclusion}

Mobile terminal technology attracts a wide range of applications with its huge performance advantages. In the power system, which has a wide application prospect in energy meter reading, transmission line condition monitoring, electric bill payment and mobile field operation.For this reason, we have developed a emergence repair system based on mobile terminals, .We also following the principle of appropriate protection design the system's security mechanism for the safety of mobile Internet technology defects. We hope to bring relevant applications to learn from. Mobile Internet security is a complex systemic problem, involving terminal equipment, mobile networks, business applications and other aspects of the problem. Lack of security on any one of these areas poses a serious threat to the overall security of mobile applications. As a result, the security mechanisms necessary for the application are essential to ensure that the system operates in a stable and safe manner.

\section{Acknowledgement}

In this paper, the research was sponsored by the National Natural Science Foundation of China (Project No. 61300040) and Scientific Research Project of Hebei Province (Project No. Z2012077)

\section{References}

[1] Wang Fayi, Xu Tongyu, Li Jinghong, Development and application of electrical emergency repair system. Rural Electrification,2011,287(4),45-46. 
[2] He Yan, Liu Yihang, Design and implementation of real-time electrical repair service system based on PDA. Fujian Computer, 2011,498-99,152.

[3] Feng Fang, Zhang Xingzhong, Wang Lihui, Research of electrical repair scheduling system based on GPS/GPRS/GIS.Computer Measurement \& Control,2013,21(2),526-528.

[4] Lian Huaqing, Yang Lida, Wang Jijun, Cheng Weihua, Research and design of electrical perair system based on GIS. Electronics World 2014,16,175-176.

[5] Lu Shida, Research and application of SSL in constructing secure smart grid. Computer Applications and Software,2012,6,282-284.

[6] Chen Lu, Chen Huazhi, Deng Song, Zhang Tao, Ma Yuanyuan, Research on security access control method of electric intranet terminal. Electric Power ICT, 2014,12(6),1-4.

[7] Wang Chun-XIN, Li Xin, Yu Ran, Yi Ping, Attack and defense research on mobile intelligent terminals used in electrical systems. Netinfo Security,2014,4,70-77.

[8] Dong Liang, Li Jing-lin, Zhou Lei, Zhan Wei, Yuan Hui, Peng Xiang-li, Mobile terminal security access system construction practice of Hubei Electric Power Company. Hubei Electric Power,2015,6,64-66. 